\title{
Distortion Rejecting Magneto-Inductive 3-D Localization (MagLoc)
}

\author{
Traian E. Abrudan, Member, IEEE, Zhuoling Xiao, Student Member, IEEE, Andrew Markham, and Niki Trigoni \\ Department of Computer Science, University of Oxford \\ E-mails: firstname.lastname@cs.ox.ac.uk
}

\begin{abstract}
Localization is a research area that, due to its overarching importance as an enabler for higher level services, has attracted a vast amount of research and commercial interest. For the most part, it can be claimed that GPS provides an unparalleled solution for outdoor tracking and navigation. However, the same cannot yet be said about positioning in GPSdenied or challenged environments, such as indoor environments, where obstructions such as floors and walls heavily attenuate or reflect high frequency radio signals. This has led to a plethora of competing solutions targeted towards a particular application scenario, yielding a fragmented solution landscape. In this paper, we present a fresh approach to 3-D positioning based on the use of very low frequency $(\mathrm{kHz})$ magneto-inductive (MI) fields. The most important property of MI positioning is that obstacles like walls, floors and people that heavily impact the performance of competing approaches are largely "transparent" to the quasi-static magnetic fields. MI has a number of challenges to robust operation that distort positions, including the presence of ferrous materials and sensitivity to user rotation. Through signal processing and sensor fusion across multiple system layers, we show how we can overcome these challenges. We showcase its highly accurate 3-D positioning in a number of environments, with positioning accuracy below $0.8 \mathrm{~m}$ even in heavily distorted areas.
\end{abstract}

Index Terms-Localization, Indoor, Magnetic fields, Magnetic modulators, Distortion, Design.

\section{INTRODUCTION}

$\mathbf{I}$ $\mathrm{N}$ this paper, we present a fresh approach to positioning based on a technology that is inherently suited to the problem of operation in complex and cluttered environments. Key to this is the use of very low frequency $(\mathrm{kHz})$ magnetoinductive (MI) vector fields that allow for 3-D positioning between a single transmitter-receiver pair. Secondly, the most important property of MI positioning is that obstacles like walls, floors and people that heavily impact the performance of high frequency radio, sound and light based location approaches are largely "transparent" to the quasi-static magnetic fields. In addition, due to the extremely long wavelengths $(\mathrm{km})$, multipath is not a concern. Thus, the inherent properties of these fields neatly solve the two main challenges (attenuation and multipath) faced by existing technologies.

Put together, these features of 3-D single transmitter positioning and excellent signal penetration result in a shift in the conventional approach to installing a positioning system,

Manuscript received August 15, 2014; revised December 5, 2014, January 21, 2015; accepted February 10, 2015. The authors would like to thank EPSRC for funding this research (Grant ref. EP/L00416X/1 Digital Personhood: Being There: Humans and Robots in Public Spaces (HARPS)). commanding a unique vantage point. The current approach to positioning is to install a number of devices, typically spread within the area of interest, elevated to provide good coverage. For most systems, a minimum of four non-colocated positioning devices are required to provide 3-D positioning. In many ways, the user has to place positioning devices in locations which are desirable from the point of view of the location system, rather than convenient from the user's standpoint. The perspective we adopt is that a positioning system should be a service, invisible, hidden away in a cupboard or a basement, just like any other service like heating or plumbing.

Although MI has a number of advantages that set it apart from existing techniques, it also presents some unique challenges that require careful system level design, from the physical through to the application layer. We believe that these challenges prevent its widespread use and adoption. In particular, real systems suffer from two major sources of distortion. The first is caused by ferrous materials in the environment which act to bend and concentrate flux lines. This leads to inaccurate position estimation. To address this, we present a novel technique to automatically detect these distortions based on the measured channel and reject position estimates with large errors. The second source of distortion is due to user dynamics, as rotation of the receiver smears the recovered magnetic signal. With the aid of short-term inertial measurements, we compensate for change in the receiver's orientation whilst receiving a transmission. Furthermore, we emphasize that unlike many sensor fusion localization techniques in the literature using Bayesian filters, we employ fusion at multiple functional layers such as: raw magnetic signal rotation correction using inertial data, automatic sensor selection depending on the environment, and particle filtering in the final stage. In particular, the following specific contributions are made:

1. Full design, implementation and optimization of a robust magneto-inductive positioning system

2. A rotation stabilization technique to compensate for receiver rotation, and appropriately correct the distorted magnetic signals using inertial measurements

3. We propose a novel method to automatically detect field distortions due to the environment and switch to an appropriate operation mode

4. Using a single transmitter, in outdoor and many undistorted indoor environments, we demonstrate 3-D positioning with a mean accuracy of $0.3 \mathrm{~m}$, over a $20 \mathrm{~m}$ diameter 


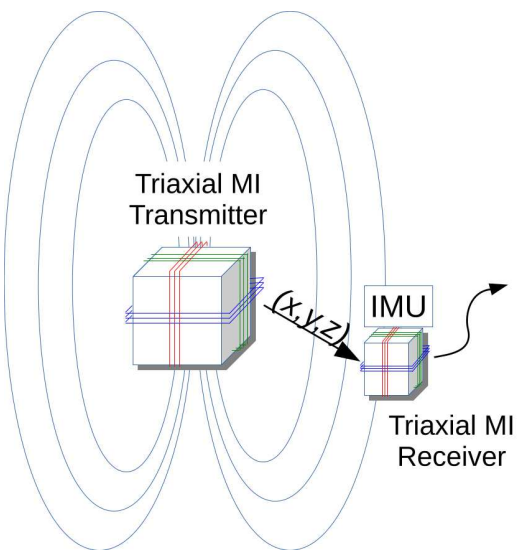

Fig. 1. The MagLoc system: A triaxial transmitter generates low frequency magneto-inductive fields which can be arbitrarily oriented. A mobile MI receiver detects these fields, and with the aid of an IMU, determines accurate 3-D positions.

sphere

5. When operating in heavily distorted environments, we can attain mean positioning accuracy below $0.8 \mathrm{~m}$ through the use of our multi-level fusion approach

The rest of our paper is organized as follows: Section II presents the high-level architecture of the proposed MagLoc system. Section III describes the multiple-input multipleoutput (MIMO) magnetic channel. The related signal processing algorithms are provided in Section IV. In Section V, we show how 3-D localization can be achieved in different environments. Section VI describes our hardware/software implementation used to obtain the experimental results. Section VII provides extensive experiments carried out in five different environments, one outdoor, and four indoor. A survey of the related work is provided in Section VIII. Section IX concludes the paper.

\section{MagLoc System Architecture}

The overall objective is to develop a robust 3-D positioning system that is able to operate in a number of challenging environments with sub-meter accuracy. The MagLoc system itself is shown in Fig. 1. It comprises a triaxial transmitter (TX) which generates low frequency magneto-inductive (MI) fields which can be arbitrarily oriented in 3-D space, using three orthogonal coils. The mobile receiver has triaxial receiving coils which detect the weak quasi-static magnetic fields and determines, with the aid of an inertial measurement unit (IMU), the position of the receiver.

In this section, we provide an overview of the main system components, and their functional role. In particular, we optimize the signal processing chain in the receiver in order to reliably extract weak signals, in the presence of background noise. The high-level receiver (RX) architecture is shown in Fig. 2. It contains three main components (corresponding to the light-green boxes in the figure): Sensors block, MI signal processing block, and Localization block.

Within the Sensors block (see Fig. 2), three mutually orthogonal coils act as MI sensors measuring a 3-D-modulated magnetic vector field in a particular location. To cope with receiver dynamics, an inertial measurement unit (IMU) is also incorporated within the sensors block, which serves three main purposes. i) Rotation stabilization: The IMU provides a stable $\mathrm{RX}$ navigation frame in the short term over a few seconds, such that the magnetic signals sensed in each RX coil are unaffected by the fast changes in the user orientation during the transmission of a frame. ii) Sanity check of the MI-based estimated 3-D position displacements over short time periods; iii) Tracking the receiver position over longer time periods using a particle filter; we emphasize the fact that the sensor fusion is done at three different functional layers, not just in the final filtering stage, as commonly done in the literature.

The MI signal processing block (see Fig. 2) contains three different sub-blocks: Rotation stabilization, 3-D correlator, and Channel matrix estimation sub-block. The MI signals are passed through a correlator (Fig. 2) which uses short, low power codes for close range navigation and long, high power codes for long distance positioning. Based on the correlator output, the channel matrix is estimated along with the corresponding magnetic RSSI (received signal strength indicator).

The Localization block (see Fig. 2) consists of several sub-blocks which will be explained in detail throughout the paper. The novel distortion detection sub-block automatically determines whether the environment acts similarly to freespace, or if it acts to distort the magneto-inductive field. Note that even in metal rich environments, not all measurements are distorted. This property allows us to use sporadic non-distorted 3-D position estimates to calibrate and compensate the system. Based on its decision, the system switches to an appropriate operation mode: 1) "undistorted environment" (lower branch in a white box in Fig. 2), or 2) "distorted environment" (upper branch in a white box in Fig. 2). The two operation modes are briefly described below.

1) In an undistorted environment, the channel matrix can be directly used to derive the precise 3-D position of the receiver. However, in order to double check the reliability of the position, an IMU-based sanity check is included i.e., to determine whether the relative motion predicted by the inertial data over a few seconds is similar to the one predicted by the magnetic data.

2) In order to make the system more flexible, it also needs to operate in metal rich environments such as inside reinforced steel buildings. It must be noted that although an environment may frequently distort the channel, there are sporadic locations where distortion is low and these can be used as periodic 3-D "anchors" to compensate for IMU drift. Although the elements of the channel matrix may be distorted, as large metal objects act to concentrate the flux lines, changing their direction, the relationship between range and energy can be accurately modeled as a logarithmic relationship. The range estimates are still very reliable, as it will be shown later.

A particle filter (PF) is used in the final fusion stage in order to track the user's location. When the channel conditions are poor, the PF fuses magneto-inductive RSSI-based range estimates with inertial data, otherwise it fuses full 3-D position estimates with inertial data. 


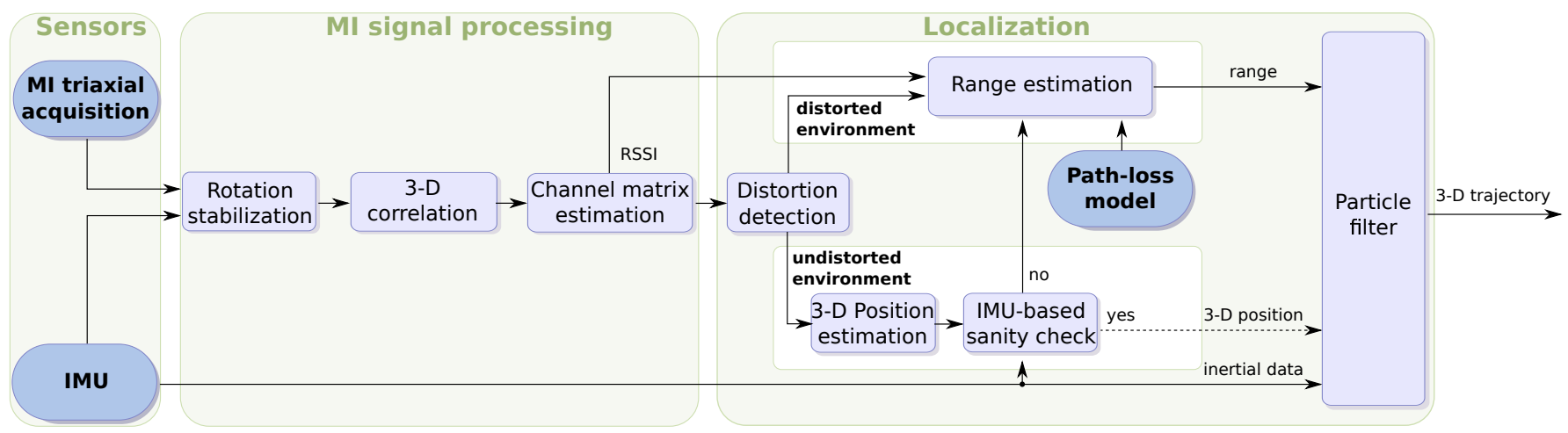

Fig. 2. Receiver architecture design, showing how MI and IMU data are processed in order to achieve real-time 3-D tracking.

\section{Magneto-Inductive Channel}

In this section, we introduce the 3-D channel model, which is used to predict the magnetic field at a particular location, under the assumption of operation in a distortion-free environment. We apply the magnetic dipole equations [1] to a TX-RX pair equipped with three mutually orthogonal coils (for more details, see also [2]). Consider a triaxial transmitter located at the origin of a North-East-Down coordinate system, $(x, y, z)=[0,0,0]^{T}$. Let the triaxial RX position in 3-D be described by the position vector $\mathbf{r}=\left[x_{r}, y_{r}, z_{r}\right]^{T}$ in the same TX frame. The TX-RX range is $r=\|\mathbf{r}\|_{2}$. TX is sequentially energized in each axis, i.e., a signal is transmitted on the $X$-axis, then the $Y$, and finally, the $Z$. The corresponding magnetic moments are:

$$
\mathbf{m}_{i}=N_{\mathrm{TX}} I_{\mathrm{TX}} A_{\mathrm{TX}} \mathbf{e}_{i},
$$

where $N_{\mathrm{TX}}$ is the number of turns of the transmitter coil, $I_{\mathrm{TX}}$ is the transmitter coil current, $A_{\mathrm{TX}}$ is the cross-sectional area of the transmitter coil, and $\mathbf{e}_{i}, i=1,2,3$ are the excitation versors corresponding to the standard Euclidean basis vectors. Given an arbitrary magnetic moment $\mathbf{m}$, the magnetic flux density at an arbitrary position $\mathbf{r}$ can be described in the TX frame using the magnetic dipole equations [1]:

$$
\mathbf{B}(\mathbf{r}, \mathbf{m})=\frac{\mu_{\mathrm{TX}}}{4 \pi}\left[\frac{3 \mathbf{r}\left(\mathbf{m}^{T} \mathbf{r}\right)}{r^{5}}-\frac{\mathbf{m}}{r^{3}}\right]=\frac{\mu_{\mathrm{TX}}}{4 \pi r^{3}}\left[\frac{3 \mathbf{r r}^{T}}{r^{2}}-\mathbf{I}_{3}\right] \mathbf{m},
$$

where $\mu_{\mathrm{TX}}$ is the magnetic permeability of the TX coil core, $\mathbf{I}_{3}$ is the $3 \times 3$ identity matrix, and $(\cdot)^{T}$ denotes the matrix transpose operation. For each value of the magnetic moment $\mathbf{m}_{i}, i=1,2,3$ in Eq. (1), we obtain a vector $\mathbf{b}_{i}=\mathbf{B}\left(\mathbf{r}, \mathbf{m}_{i}\right)$, still expressed in the transmitter frame.

Let $\Omega \in S O(3)$ be an orthogonal matrix describing the orientation of the receiver frame with respect to the transmitter frame. Grouping the three column vectors $\mathbf{b}_{i}$ in a matrix $\mathbf{B}_{1,2,3}=\left[\mathbf{b}_{1}, \mathbf{b}_{2}, \mathbf{b}_{3}\right]$, the magnetic vector field can be described in the receiver frame as:

$$
\boldsymbol{\Omega B}_{1,2,3}=\frac{\mu_{\mathrm{TX}} N_{\mathrm{TX}} I_{\mathrm{TX}} A_{\mathrm{TX}}}{4 \pi r^{3}} \boldsymbol{\Omega}\left[\frac{3 \mathbf{r r}^{T}}{r^{2}}-\mathbf{I}_{3}\right] \underbrace{\left[\mathbf{e}_{1}, \mathbf{e}_{2}, \mathbf{e}_{3}\right]}_{\mathbf{I}_{3},}
$$

The vector of voltages induced in each of the three RX axes due to the excitation $\mathbf{e}_{i}$ at $\mathrm{TX}$ is

$$
\mathbf{v}_{i}=2 \pi f \mu_{\mathrm{RX}} N_{\mathrm{RX}} A_{\mathrm{RX}} \boldsymbol{\Omega} \mathbf{b}_{i},
$$

where $f$ is the frequency of the excitation, $\mu_{\mathrm{RX}}$ is the magnetic permeability of the coil core, $N_{\mathrm{RX}}$ is the number of turns, and $A_{\mathrm{RX}}$ is the area of the $\mathrm{RX}$ coil. The input excitation corresponds to a space-time code whose coding matrix is $\mathbf{I}_{3}$ (see Eq. (3)). Therefore, we can define the $3 \times 3$ MIMO channel matrix whose $i$ th column is the vector $\mathbf{v}_{i}$ in Eq. (4), as follows:

$$
\mathbf{S} \triangleq\left[\mathbf{v}_{1}, \mathbf{v}_{2}, \mathbf{v}_{3}\right]=2 \pi f \mu_{\mathrm{RX}} N_{\mathrm{RX}} A_{\mathrm{RX}} \boldsymbol{\Omega B}_{1,2,3} .
$$

The element $s_{j, i}$ of matrix $\mathbf{S}$ (which from now on we will call the channel matrix) describes the voltage induced from the $i$ th TX coil to the $j$ th RX coil. It can be seen that the detected signals are a function not only of the relative location between transmitter and receiver, but also the relative orientation between the transmitter and receiver frames. However, note that the orientation of the receiver is an orthogonal transform, i.e. it preserves the Frobenius norm (overall power) in the signal. We show later that the channel Gram matrix defined as

$$
\mathbf{C} \triangleq \mathbf{S}^{T} \mathbf{S}
$$

is orientation invariant, and in an undistorted environment, can be used to estimate the 3-D position.

\section{MI Signal Processing}

In this section, we explain the MI signal processing block (see Fig. 2) and its related estimation techniques. We follow a similar approach to magnetic vector modulation in [3], where each TX axis generates a BPSK encoded signal sequentially. However, unlike this approach, we do away with the initial preamble and carrier tone and treat the message in its entirety as a 3-D magnetic vector signal to be correlated, i.e., each symbol is now regarded as a "chip" instead of a bit. By not treating each axis separately, we are able to yield gains in detecting weak signals as the loss of a single bit in the frame will not cause the loss of the whole frame. Equally well, if a single axis, either on the transmitter or receiver, is subject to strong background noise, it is still possible to recover the frame from the remaining two axes, allowing for estimation of range even in noisy environments. In this section, we first describe the novel rotation stabilization technique, followed by the correlation estimator and lastly the channel matrix estimation. To allow a multi-transmitter operation, we employ a hybrid CDMA/FDMA transmission. 


\section{A. IMU-based Rotation Stabilization}

One of the issues of using a correlation decoder is that it relies on the channel maintaining coherence over the period of the signal to be correlated. Although the channel itself is virtually stationary and is not subject to fading due to the absence of multipath, rotation of the receiver whilst receiving a transmitter frame causes the signal to smear among the receiver axes, as Eq. (3) shows. This can result in poor channel estimates, as energy will be shared between channels over the duration of the transmission. The Gram matrix $\mathbf{C}$ (6) is orientation invariant as long as the channel remains stationary over the frame duration. However, the RX rotation perturbs the off-diagonal coefficients of $\mathbf{C}$, which are estimated based on the entire packet and used to derive the 3-D position. More problematic is if the rotation results in a phase reversal of the carrier, as this will garble the channel estimation, both in terms of magnitude, but also in terms of relative sign. Thus, although using correlation decoding provides processing gain, boosting weak signal detection, it requires that the receiver is kept stationary over the transmission. This is undesirable from the perspective of providing responsive and accurate mobile tracking. To tackle this issue, we exploit the on-board IMU to perform a rotational correction over the duration of the transmitter frame. As frames are transmitted at equal time intervals, it is possible to learn the timing using a delay-lockedloop (DLL), to indicate the expected time instant of the start of a frame. Over a short-time period (up to a few seconds), an IMU can provide an excellent estimate of its orientation relative to a starting point, before drift starts to become a dominating concern. Informed by the DLL, at the predicted start of the frame the orientation is set to identity matrix. Note that this is not the absolute orientation of the sensor frame in global coordinates, as this would require accurate measurements of compass heading which is notoriously inaccurate when operating in metal rich environments. A Kalman Filter is then used to estimate the subsequent rotation of the sensor frame, relative to the starting orientation, outputting a matrix $\boldsymbol{\Omega}_{n}$ at each symbol time instance $n$. This is then used to correct the received voltage vectors $\mathbf{v}_{i}$ (4), shifting each measurement back to a stable reference frame, i.e., $\boldsymbol{\Omega}_{n} \mathbf{v}_{i}$.

Fig. 3 shows the fluctuations of off-diagonal coefficients of the Gram matrix $\mathbf{C}=\mathbf{S}^{T} \mathbf{S}$ before and after the user rotation compensation. The magnitude of coefficients $c_{i, j}$ of $\mathbf{C}$ decays with the inverse 6th power of distance, thus exhibiting a very high dynamic range. For illustrative purposes, we compress their range as follows: $C_{i, j}=\operatorname{sign}\left(c_{i, j}\right)\left|c_{i, j}\right|^{1 / 6}, i \leq j \leq 3$. We may notice in Fig. 3 that the variation of the coefficients has been reduced. In our experiment, the receiver was kept at a fixed location while performing two full rotations on the $(x, y)$-plane. We would like to point out that $C_{i, j}$ may also serve as rotation invariant fingerprints.

\section{B. 3-D Signal Correlation}

Typical indoors sources of electromagnetic noise include electric appliances (monitors, air conditioning, lighting equipment, microwave oven, vacuum cleaner, etc.) that generate
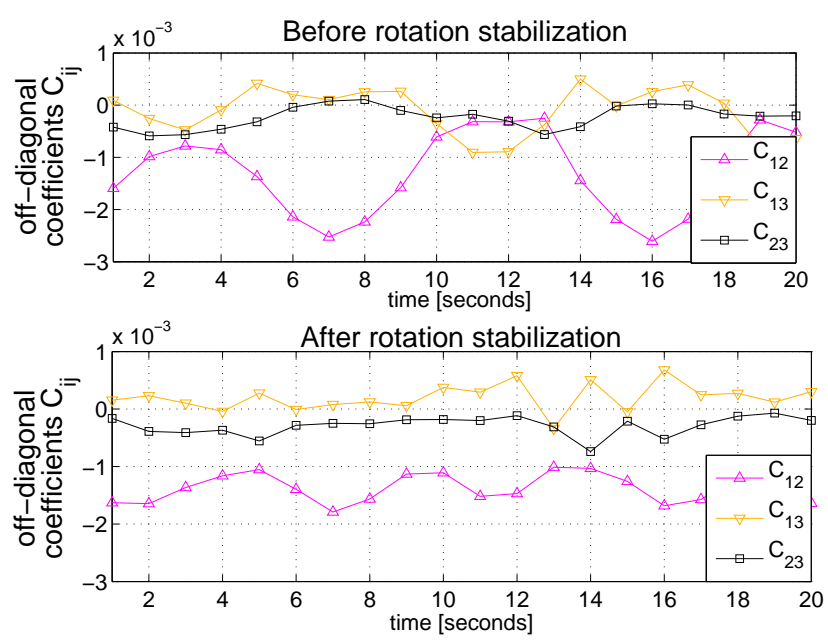

Fig. 3. IMU-based rotation stabilization. The elements of $\mathbf{C}$ are invariant w.r.t. user orientation if and only if the channel remains constant during one frame. Otherwise, the rotation will cause a periodic fluctuations which need to be corrected.

high-order harmonics. Due to the fast field decay, the interference decreases significantly already one meter away from the source [4]. We also observed experimentally that the generated harmonics are unnoticeable around the carrier frequency of $2.5 \mathrm{kHz}$, this being one of the reasons why we chose this operating frequency. Given a certain level of background noise, there are two ways to increase detection range - transmit the same signal using a greater power or transmit at the same power output for a longer period. We adopt the latter approach, using 3-D direct sequence spread spectrum (DSSS) coding to provide processing gain, transmitting chips on different transmitter axes, much like MIMO diversity. The major difference with traditional MIMO is that the channel is purely real and does not have any multipath, due to near-field quasi-static operation [5]. In practice, the dynamic range of the signal is such that saturation occurs nearby the transmitter. In order to overcome this challenge, we use two different power levels within the same transmitted frame. The correlator is a matched filter and a maximum likelihood estimator. In addition, by broadening the spectrum, the impact of narrowband interferers, such as mains harmonics (e.g. near power transformers) is greatly reduced. In extreme EM interference environments, such as transforming power stations, special care in choosing the carrier frequency may be required. Our software defined magnetic transceiver provides flexibility in terms of carrier frequencies and modulation techniques, thus enabling application-specific designs.

\section{Channel Matrix Estimation}

Given a set of received signals corrupted by noise, the aim of this block is to provide an accurate estimation of the channel between the transmitter and receiver. Let $\mathbf{P}_{\mathrm{TX}}$ be a known $N \times 3$ sequence transmitted over the three transmitter axes, and $\mathbf{P}_{\mathrm{RX}}$ its received version corrupted by Gaussian noise (we have verified that this assumption holds). The least-squares estimate of the channel matrix (which in this case is also 
Maximum Likelihood estimate) is

$$
\hat{\mathbf{S}}=\left[\mathbf{P}_{\mathrm{TX}}^{\dagger} \mathbf{P}_{\mathrm{RX}}\right]^{T},
$$

where $(\cdot)^{\dagger}$ denotes the Moore-Penrose pseudoinverse.

\section{LOCALIZATION}

In this section, we explain the Localization block in Fig. 2, and its main sub-blocks. The novelty of our system is that we fuse the MI and IMU sensors at various functionality layers, and not just in the filtering stage, as commonly done in the literature. First, the inertial data is used to correct the raw magnetic signal affected by the user's change of orientation during the frame transmission. Second, we automatically detect the environment distortions, and discard unreliable data. A sanity check compares the estimated position displacements from MI and IMU measurements to ensure reliable measurements. Depending on these decisions, we treat the sensor outputs differently. Thirdly, these outputs are fed to the final fusion stage which undertakes traditional filtering and fusion, in order to estimate the user's trajectory. We first discuss our novel distortion detection technique which predicts the reliability of the 3-D position estimates. If low distortion is detected, then a full 3-D position is computed. However, if high distortion is detected, then the TX-RX range is used instead. Lastly, the fusion block integrates measurements from the IMU and the MI to determine an accurate position of the receiver.

\section{A. Distortion detection}

Distortion is introduced by metallic objects which alter the spatial distribution of the magnetic field. This manifests as a shift in the relative energy among axes, as compared to the free-space. The purpose of this sub-block is to determine whether a redistribution of energy has indeed taken place. It should be noted that although an environment may be distorting in general, it typically does not distort all measurements and there are sporadic positions where distortion is negligible. In order to select the appropriate operation mode of the system, it is necessary to automatically detect whether or not the receiver is operating within a distorted environment. Based on Eqs. (3) and (5), we can write

$$
\mathbf{S}=\underbrace{c}_{\text {range-dependent }} \underbrace{\Omega\left[\frac{3 \mathbf{r r}^{T}}{r^{2}}-\mathbf{I}_{3}\right]}_{\text {range-independent }} .
$$

where $c \triangleq \mu_{\mathrm{TX}} \mu_{\mathrm{RX}} N_{\mathrm{TX}} N_{\mathrm{RX}} I_{\mathrm{TX}} A_{\mathrm{TX}} A_{\mathrm{RX}} f /\left(2 r^{3}\right)$ is a scaling factor that is inversely proportional to the cube of the range (see Eqs. (3) and (4)). With this definition of $c$, we are able to separate Eq. (8) in range dependent and independent parts. Since the range $r=\|\mathbf{r}\|$, the factor $\Omega\left[3 \mathbf{r r}^{T} / r^{2}-\mathbf{I}_{3}\right]$ is range-independent (only depends on the 3-D direction of $\mathbf{r}$, not on its norm). We first get rid of the arbitrary RX orientation $\Omega \in S O(3)$ as follows. Let us consider the channel Gram matrix $\mathbf{C}=\mathbf{S}^{T} \mathbf{S}$. Using Eq. (8), it can be seen that

$$
\mathbf{C}=c^{2}\left[\frac{3 \mathbf{r} \mathbf{r}^{T}}{r^{2}}-\mathbf{I}_{3}\right]^{T} \underbrace{\boldsymbol{\Omega}^{T} \boldsymbol{\Omega}}_{\mathbf{I}_{3}}\left[\frac{3 \mathbf{r} \mathbf{r}^{T}}{r^{2}}-\mathbf{I}_{3}\right],
$$

and that the sensor orientation has been removed. In an undistorted environment, Eq. (9) is satisfied with high fidelity. In distorted environments, we do not require this equation to be satisfied. Instead, we exploit it in order to detect whether the field is distorted or not. The orientation invariance we are interested in holds in any environment, for arbitrary orientations of the sensor frame. In the case of undistorted signals, the full channel matrix $\mathbf{S}$ may be used directly to estimate the 3-D position, as it will be shown in Section V-B. Recall that the MIMO channel matrix $\mathbf{S}$ in Eq. (5) describes the voltages induced from the TX triaxial coil into the RX triaxial coil. Looking at the Gram matrix $\mathbf{C}=\mathbf{S}^{T} \mathbf{S}$ from an energy transfer perspective, the quantity trace $\{\mathbf{C}\}=\|\mathbf{S}\|_{\mathrm{F}}^{2}$ corresponds to the total energy received by all receiver coils, from all transmitter coils. In Eq. (9), notice that the eigenvalues of $\mathbf{C}$ are $\left\{4 c^{2}, c^{2}, c^{2}\right\}$ (the maximum eigenvalue is four times larger than the remaining two, as expected from the dipole equation). In order to get rid of the range-dependent scaling factor $c$, we divide by the average of the eigenvalues (which is $\operatorname{trace}\{\mathbf{C}\} / 3=2 c^{2}$ ), and we obtain the following scaled eigenvalue vector

$$
\boldsymbol{\lambda}=\left[2, \frac{1}{2}, \frac{1}{2}\right]^{T}
$$

In an undistorted environment, the vector $\boldsymbol{\lambda}$ remains constant regardless of the locations and orientations of transmitter and receiver. Therefore, any deviations from this may be used to indicate the presence of distortions in the environment. Distortions are caused by large amounts of metal, typically steel mesh used in concrete reinforcement, which causes the channel model to become inaccurate at predicting the received signals. In the presence of metallic objects, the coupling of each transmitter coil to each receiver coil is different compared to an undistorted environment. Consequently, the energy transfer matrix $\mathbf{S}$ may differ substantially. The different mutual coupling is reflected in how the eigenvalues of $\mathbf{C}$ are spread, i.e., how the energy has "moved" from one axis to another. In order to quantify the change in the eigenvalues spread, we define the following criterion that penalizes the departure of the estimated scaled eigenvalue vector $\hat{\lambda}$ from the theoretical free-space value $\boldsymbol{\lambda}$ given in Eq. (10)

$$
\mathcal{J}(\hat{\boldsymbol{\lambda}})=\frac{\|\hat{\boldsymbol{\lambda}}-\boldsymbol{\lambda}\|_{2}}{\|\boldsymbol{\lambda}\|_{2}}
$$

where $\hat{\lambda}$ corresponds to the eigenvalues of estimated Gram matrix $\hat{\mathbf{C}}=\hat{\mathbf{S}}^{T} \hat{\mathbf{S}}$ sorted in descending order, and divided by their average. If $\mathcal{J}$ (Eq. (11)) exceeds a predefined threshold $a$, then the environment is considered to be distorted. A suitable threshold can easily be determined experimentally by taking position measurements in various environments, but values of $a<0.1$ generally show good performance. Alternatively, a soft decision can be used to inform the probabilistic fusion stage.

\section{B. Operation in low distortion conditions}

Many environments have low concentrations of ferrous material and in these cases, the theoretical model of the magnetic field vector distribution very closely agrees with real 
measurements. These environments include outdoors, underground, underwater, and some indoor environments. In these cases, the channel matrix contains sufficient information to uniquely position the receiver in $3-\mathrm{D}$, subject to a hemispherical ambiguity. This is due to the inherent symmetry of the magnetic fields, leading to two possible estimated positions $\left(\hat{x}_{r}, \hat{y}_{r}, \hat{z}_{r}\right)$, or $\left(-\hat{x}_{r},-\hat{y}_{r},-\hat{z}_{r}\right)$ [2, Ch. 13]. This can easily be removed at the application layer, with the aid of a map, inertial measurements or signals from two (or more) transmitters. In this section, we show how magneto-inductive positioning can be used to derive the position of a triaxial receiver in 3-D, using signals from a triaxial transmitter.

1) Range Estimation : The latter factor in Eq. (8) depends only on the versor $\mathbf{r} /\|\mathbf{r}\|$ and therefore, the Frobenius norm of $\mathbf{S}$ defines the received power, which in free-space, is proportional to the inverse cube of the range, i.e., $\|\mathbf{S}\|_{\mathrm{F}} \propto r^{-3}$. Since rotations preserve the Frobenius norm, using triaxial coils both at TX and RX makes the range invariant w.r.t. the relative orientation of TX and RX. We define the overall RSSI (Received Signal Strength Indicator) measured in $\mathrm{dB}$ as

$$
\rho \triangleq 20 \log _{10}\|\mathbf{S}\|_{\mathrm{F}} .
$$

The law describing the RSSI vs. distance in free-space is

$$
\rho=\rho_{0}-60 \log _{10}\left(r / r_{0}\right),
$$

where $\rho_{0}$ is the RSSI measured at some reference distance $r_{0}$, and can be determined by calibration. Thus, the range between transmitter and receiver can be estimated as

$$
\hat{r}=r_{0} 10^{\left(\rho_{0}-\rho\right) / 60} \text {. }
$$

Since the near-field channel model is characterized and dominated by an inverse cube roll-off, the field strength decays at a rate of $60 \mathrm{~dB} /$ decade [5] i.e. a ten-fold increase in distance results in a thousand-fold reduction in signal strength. For some applications, such as secure communication, the rate of attenuation is a desirable property. For positioning, rapid decay is a mixed-blessing. On the one hand, it limits the operation range, which requires careful signal and hardware design. On the other hand, the steep relationship between strength and distance means that a small change in distance results in a large and measurable change in signal strength, yielding high accuracy. This is demonstrated by our experiment in Section VII-B.

2) 3-D position estimation : In order to determine the position vector $\mathbf{r}$, we propose an elegant matrix-based technique that exploits the rank-one term $\mathbf{r r}^{T} / r^{2}$ in Eq. (8). The modulus $r=\|\mathbf{r}\|$ (the range) has been estimated by now (Eq. (14)), we only need to determine the direction of $\mathbf{r}$ in $3-\mathrm{D}$. Let $\mathbf{C}=\mathbf{U D U}^{T}$ be the eigendecomposition of the Gram matrix (6). From Eq. (8), we obtain

$$
\frac{\mathbf{r}}{\|\mathbf{r}\|} \frac{\mathbf{r}^{T}}{\|\mathbf{r}\|}=\frac{1}{3 c} \underbrace{\mathbf{C}^{1 / 2}}_{\mathbf{U D}^{1 / 2} \mathbf{U}^{T}}+\frac{1}{3} \underbrace{\mathbf{I}_{3}}_{\mathbf{U U}^{T}}=\mathbf{U}\left[\frac{1}{3 c} \mathbf{D}^{1 / 2}+\frac{1}{3} \mathbf{I}_{3}\right] \mathbf{U}^{T}
$$

which is a rank-one matrix. Consequently, the position versor $\mathbf{r} /\|\mathbf{r}\|$ we are interested in corresponds to the maximal eignevector $\hat{\mathbf{u}}_{\max }$ of the estimated Gram matrix $\hat{\mathbf{C}}$. Finally the 3-D position vector can be written as

$$
\hat{\mathbf{r}}=\hat{r} \hat{\mathbf{u}}_{\max } .
$$

\section{IMU-based sanity check}

In the short term (over a few seconds), the IMU provides very reliable estimates of the position displacement. We take advantage of this property in order to detect occasional failures of the distortion detection algorithm, although the failure rate is very low (as it will be seen in experiments). When the displacement predicted by the MI-based positioning deviates significantly from the displacement predicted by the inertial sensors, we rely on the latter measurements. This way, we prevent these bad position estimates from propagating through to the final fusion stage $(\mathrm{PF})$. Note that we still use the range measurements, as these remain reliable.

\section{Operation in Distorted Environments}

When operating in environments characterized by an abundance of metal, such as in typical steel-reinforced buildings, the measured and predicted magnetic fields can vary greatly. This is because metallic objects act as flux concentrators, bending the field lines and degrading overall positioning accuracy from tens of centimeters to over a meter. Thus, it is necessary to calibrate the path-loss model which relates total received signal energy to transmitter-receiver range, by modifying Eq. (12), with the following

$$
\hat{r}=r_{0} 10^{\left(\rho_{0}-\rho\right) /(10 \alpha)},
$$

where $\alpha$ is the estimated path loss exponent ( $\alpha=6$ in free-space) and $r_{0}$ is the reference (offset) level. The two values $r_{0}$ and $\alpha$ may be estimated using linear regression similarly to RF channel model. Our experiments show that MI RSSI measurements exhibit much lower variance compared to RF-based RSSI, thus much fewer measurements need to be collected to obtain a reasonable model estimation. The estimated range is used as an input to the final fusion block, which is the particle filter.

\section{E. Particle filter}

Finally, a particle filter is employed to fuse the MI measurements (range or position, depending on distortion detection) with the inertial measurements, and to track the user location over time. The likelihood of the position is inferred from the magnetic measurements, whereas the user motion is predicted from the inertial data using a pedestrian dead-reckoning (PDR) algorithm.

PDR: Our RX includes a low-cost IMU, and therefore, the double integration technique may lead to large trajectory drifts. As we assume that the device is carried by a pedestrian, we opted for an existing PDR algorithm [6] to detect the steps and the heading of the user. The displacement vector is $\Delta \mathbf{x}_{k}=$ $\left[\left(\hat{d}_{k}+\Delta d_{k}\right) \cos \left(\hat{\theta}_{k}+\Delta \theta_{k}\right),\left(\hat{d}_{k}+\Delta d_{k}\right) \sin \left(\hat{\theta}_{k}+\Delta \theta_{k}\right), \Delta z_{k}\right]^{T}$. Both the magnitude $\hat{d}_{k}$ and the angle $\hat{\theta}_{k}$ of the estimated horizontal displacement vector are assumed to be additively affected by Gaussian noises, i.e., $\Delta d_{k} \sim \mathcal{N}\left(\Delta d_{k} ; 0, \sigma_{d}^{2}\right)$, and $\Delta \theta_{k} \sim \mathcal{N}\left(\Delta \theta_{k} ; 0, \sigma_{\theta}^{2}\right)$, respectively [6], where $\sigma_{d}^{2}$ is the 
variance of the estimated horizontal displacement magnitude, and $\sigma_{\theta}^{2}$ is the variance of the heading estimates. The vertical displacement $\Delta z_{k}=0$, unless a staircase is detected (for details, see [7]). Then, $\Delta z_{k}$ equals the stair height $h_{s}$ (which can be determined from the floor map assuming that the floor height and the number of stairs are known).

Prediction: The $i$ th particle 3-D position $\mathbf{x}_{k}^{i}=\left[x_{k}^{i}, y_{k}^{i}, z_{k}^{i}\right]^{T}$ at time $k$ is updated using the estimated displacement vector $\Delta \mathbf{x}_{k}$ from the inertial measurements as: $\mathbf{x}_{k+1}^{i}=\mathbf{x}_{k}^{i}+\Delta \mathbf{x}_{k}$.

Likelihoods: The weight of each particle is determined by how much the position of this particle agrees with the magnetic path-loss model. Specifically, the weight of the $i$ th particle is determined as $w_{k}^{i}=\mathcal{N}\left(r_{k}^{i} ; \hat{r}_{k}^{p}, \sigma_{r}^{2}\right)$ where $r_{k}^{i}$ is the distance between the current particle and TX, $\hat{r}_{k}^{p}$ is the distance estimated from the magnetic RSSI using the path-loss model, and $\sigma_{r}^{2}$ is the variance of the distance estimates. When a 3$\mathrm{D}$ position estimate $\hat{\mathbf{x}}_{k}=\left[\hat{x}_{k}, \hat{y}_{k}, \hat{z}_{k}\right]^{T}$ is available, the angle between the $i$ th particle's position vector and the estimated position vector $\hat{\phi}_{k}^{i}=\angle\left(\mathbf{x}_{k}^{i}, \hat{\mathbf{x}}_{k}\right)$ is assumed to be affected by a zero-mean additive Gaussian noise. The corresponding weights are $w_{k}^{i}=\mathcal{N}\left(r_{k}^{i} ; r_{k}^{p}, \sigma_{r}^{2}\right) \mathcal{N}\left(\hat{\phi}_{k}^{i} ; 0, \sigma_{\phi}^{2}\right)$, where $\sigma_{\phi}^{2}$ is the angular variance of the 3-D position estimates. The joint likelihood function is the product of the range and angular likelihoods corresponding to all transmitters in range.

Resampling and backward belief propagation: The resampling process generates a new set of particles by replicating particles in the current set in proportion to their weights. Our particle filter also implements a backward belief propagation mechanism, which not only updates the current state, but also traces back the trajectory of a particle in the state space to improve the localization in the past [8]. This is done by replacing the history of a particle removed in the resampling stage with the history of the surviving particle after the resampling stage.

\section{IMPLEMENTATION}

This section describes our particular hardware/firmware and software implementation that was used to obtain the experimental results. The focus of the design was to be able to have a flexible platform for experimentation, and in many ways resembles Software Defined Radio (SDR). This is made especially easy by the low frequencies required.

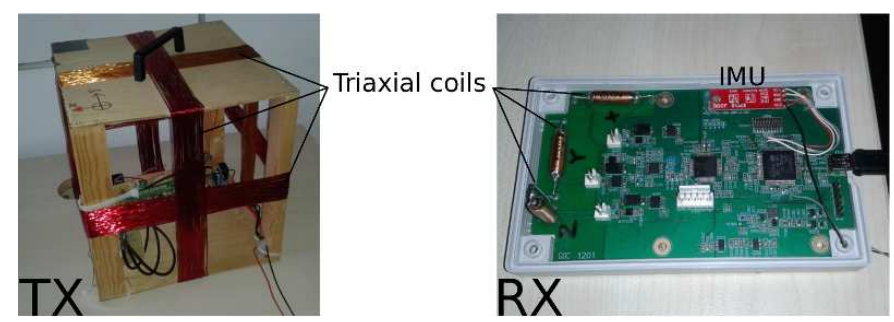

Fig. 4. Magnetic transmitter (left) and receiver (right) equipped with triaxial coils. In the RX, the three orthogonal coils (left of the board) are connected to the analog stage (center of the board), and then to the digital stage (right).

\section{A. Transmitter}

The objective of the transmitter design is to make them as simple and low-cost as possible, allowing them to be deployed easily in an area without requiring any configuration or a secondary communication channel like WiFi or Ethernet. Each transmitter is equipped with three mutually orthogonal coils, wound from $1 \mathrm{~mm}^{2}$ cross-section area insulated magnet wire wrapped on a wooden $30 \mathrm{~cm}$ cube former. Each coil consists of approximately 80 turns, and is loaded with a series capacitor. The transmitted multi-axis BPSK signal (similar to [3]) is generated by an STM32F4 micro-controller through direct digital synthesis. The three modulated signals are amplified using a $10 \mathrm{~W}$ audio amplifier (TDA2003), operating from a $12 \mathrm{~V}, 1.2 \mathrm{Ah}$ battery, or from a mains transformer. Although the peak current in the coil can easily exceed $1 \mathrm{~A}$ (due to the resonant nature of the LC circuit), the supply current of the entire TX is around $100 \mathrm{~mA}$, which is very reasonable for a static device. Each transmitter can be digitally tuned to operate over a $500 \mathrm{~Hz}$ band, centered at the chosen resonant frequency of $2.5 \mathrm{kHz}$. A symbol rate of $62 \mathrm{symbols} / \mathrm{s}$ was chosen, along with a three axis frame length of 31 symbols. TX size can be reduced without decreasing the transmission range. According to Eq. (1), we need to increase either the number of turns in the coil (more copper), or the input current (hence the power consumption). Alternatively, we may increase the magnetic permeability (e.g. using a ferrite core - see Eq. (2)). We opted for a larger, but lighter (air core) and lower-power TX device. Wiser designs are certainly possible.

We would like to point out the fact that our TX complies with the ICNIRP guidelines for limiting exposure to timevarying electro-magnetic fields [9]. The occupational RMS value at $2.5 \mathrm{kHz}$ is $1.2 \times 10^{-4} \mathrm{~T}$ which is higher than the maximum field generated by the TX coil (when user is located on-axis, touching the coil). Moreover, the fast decay of the field ensures that our TX is absolutely safe.

\section{B. Receiver}

The receiver was laid out on a 4 layer PCB, with two ground planes to reduce analog noise. The analog and digital sections were separated, each with their own ground planes. The receiver is powered by a $3.7 \mathrm{~V} 1.3$ Ah Lithium Polymer battery, which provides over 12 hours of operation. A picture of a receiver unit is shown in Fig. 4 (right), with board dimensions of $12 \times 7 \times 2 \mathrm{~cm}$. This is the third generation of highly configurable receiver, with improved noise performance over the earlier versions. The size of the receiver can be reduced to few cubic centimeters, similarly to our previous work on MI animal tracking [10]. The low noise amplifier boosts the signals from the three orthogonal coils, which are simply $12 \mu \mathrm{H}$ ferrite cored inductors, of length $18 \mathrm{~mm}$. The signals are digitized by a simultaneous sampling ADS1174I 16 bit ADC, operating in I2S mode to reduce sample jitter. The three-axis signals are then digitally down-converted with a locally synthesized carrier, performed by an ARM STM32F4 micro-controller. After filtering and decimation, frame timing is recovered with a matched filter, tuning a delay-locked loop to synchronize to the start of the frame. This information 
is used to correct the impact of changes in orientation of the receiver whilst acquiring a signal, with the aid of the Kalman filtered rotation estimations obtained from the IMU. Depending on the signal strength, either the short, low power correlation or the long, higher power correlation product is used, based on a signal strength heuristic. This is used to estimate the $3 \times 3$ channel matrix, which is transmitted to the user display (either a laptop or a mobile phone) via USB or WiFi.

\section{EXPERIMENTS}

We conducted various experiments in different environments to showcase the advantages of MI positioning, in particular its high accuracy 3-D positioning with low drift. This section includes three different types of experiments. First, we evaluate the performance of our novel distortion detection algorithm presented in Section V-A to predict large positioning errors in various environments. In the second set of experiments, we test the ranging accuracy in different environments, as well as the ability of our system to detect tiny displacements. The time stability of the magnetic RSSI in presence of moving people is also tested, and compared to the WiFi. Our last experiment investigates dynamic tracking accuracy in metallicrich environments.
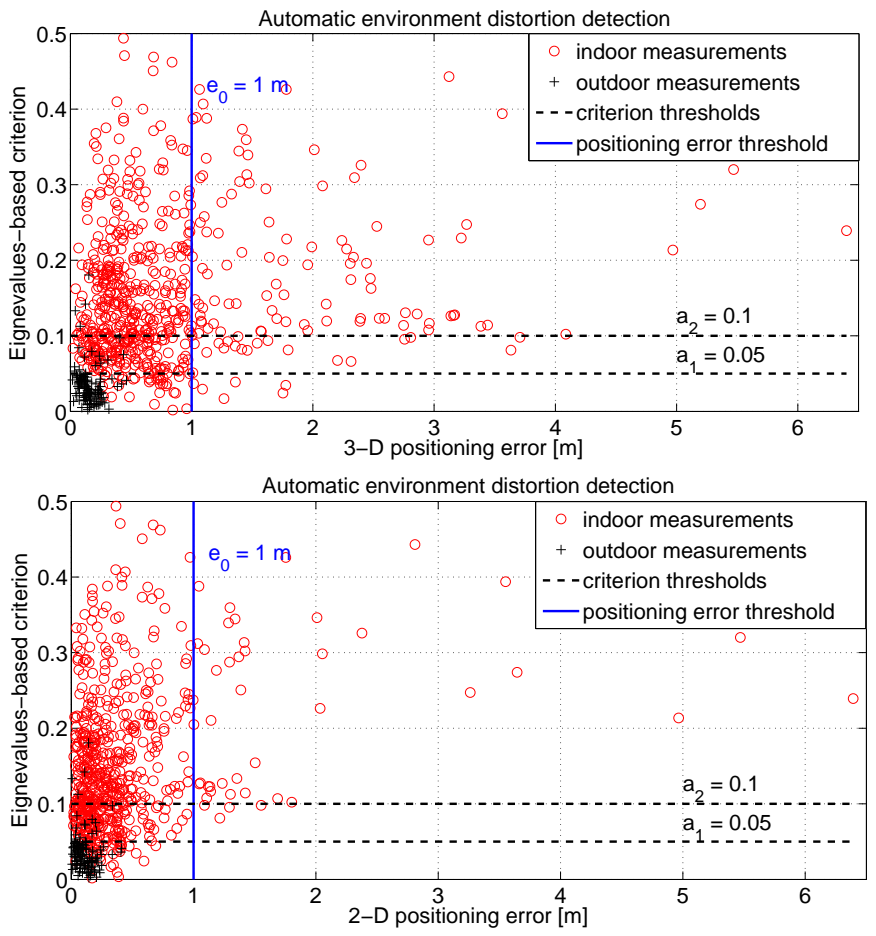

Fig. 5. Prediction of position estimation error based on the eigenvalue spread of the Gram matrix. The probability to miss larger positioning errors is significantly lower in 2-D (bottom) than in 3-D (top).

\section{A. Automatic environment distortion detection}

In this experiment, we prove the effectiveness of our novel distortion detection approach presented in Section $\mathrm{V}-\mathrm{A}$. We show the ability of the proposed eigenvalue-based criterion
$\mathcal{J}$ (11) to discriminate between undistorted and distorted environments. We selected various environments that contain heavily reinforced metallic structures structures such as: office, large lab, medium-size lab. A number of 734 measurements were collected (120 outdoor and 614 indoor). Fig. 5 (top) shows the criterion $\mathcal{J}$ (see Section V-A) versus the 3-D positioning estimation errors $e$ corresponding to the channel Gram matrix $\mathbf{C}=\mathbf{S}^{T} \mathbf{S}$. We notice in Fig. 5 (top) that the outdoor measurements (marked by black crosses) form a compact cluster, and exhibit very low values of both the criterion $\mathcal{J}$ and positioning error $e$. Most of the indoor measurements (marked by red circles) exhibit larger values of the criterion, and larger errors. Large ranging errors correspond to high values of $\mathcal{J}$. Only few points classified as "good" result in a larger positioning error. Therefore, by using a threshold $a$ that can be determined experimentally, large positioning errors can be detected with high probability. We also define a fixed threshold $e_{0}=1 \mathrm{~m}$ for the position estimation errors (submeter) above which we consider the estimates to be poor.

From now on, we will focus strictly on the measurements in distorted environments (shown by the red circles in Fig. 5 (top). At first glance, the criterion is quite "pessimistic", since there are many points that are classified as "bad" while the positioning errors are rather small. In order to show the true classification ability of our criterion, we analyze three key probabilistic metrics as follows. 1) the probability that the criterion fails to detect a large positioning error, defined as $\left.P_{\text {miss }}=\operatorname{Prob}\left[\left(e>e_{0}\right) \cup(\mathcal{J}<a)\right] 2\right)$ the false alarm rate, i.e., the probability that the criterion classifies the point to be unreliable when it actually is reliable, defined as $P_{\mathrm{FA}}=$ $\left.\operatorname{Prob}\left[\left(e<e_{0}\right) \cup(\mathcal{J}>a)\right] 3\right)$ the fraction of time that the channel matrix proved to be useful in distorted environments, defined as $P_{\text {useful }}=\operatorname{Prob}\left[\left(e<e_{0}\right) \cup(\mathcal{J}<a)\right]$.

From our experiments, in all indoor environments we have tested, the dominant component of the positioning error is along the z-axis. Fig. 5 (bottom) shows how the criterion behaves in the case of $2-\mathrm{D}$ positioning in the $(x, y)$ plane. We may notice that the overall positioning errors are smaller in 2$\mathrm{D}$ than in 3-D. There are also much fewer points corresponding to large positioning errors that exhibit low values of $\mathcal{J}$. In Fig. 5, we use two different thresholds $a_{1}, a_{2}$ for $\mathcal{J}$ that lead to different classification performance. The classification performance is summarized in Table I, for the two threshold values $a_{1}, a_{2}$, and for both for 2-D and 3-D positioning.

\begin{tabular}{|l||c|c|}
\hline Performance metric & 3-D positioning & 2-D positioning \\
\hline \hline$P_{\text {miss }}[\%] \quad\left(a_{1}=0.05\right)$ & 0.98 & 0 \\
$P_{\text {FA }}[\%]$ & 73.8 & 85.7 \\
$P_{\text {useful }}[\%]$ & 5.5 & 6.5 \\
\hline$P_{\text {miss }}[\%] \quad\left(a_{2}=0.1\right)$ & 4.1 & 0.98 \\
$P_{\text {FA }}[\%]$ & 51.0 & 60.1 \\
$P_{\text {useful }}[\%]$ & 28.3 & 31.7 \\
\hline
\end{tabular}

TABLE I

CLASSIFICATION PERFORMANCE OF THE EIGENVALUE-BASED CRITERION.

We may notice that choosing a more conservative threshold $\left(a_{1}=0.05\right)$ for the criterion $\mathcal{J}$ results in a very low value of $P_{\text {miss }}$, but unfortunately, also in a high false alarm rate $P_{\mathrm{FA}}$ 

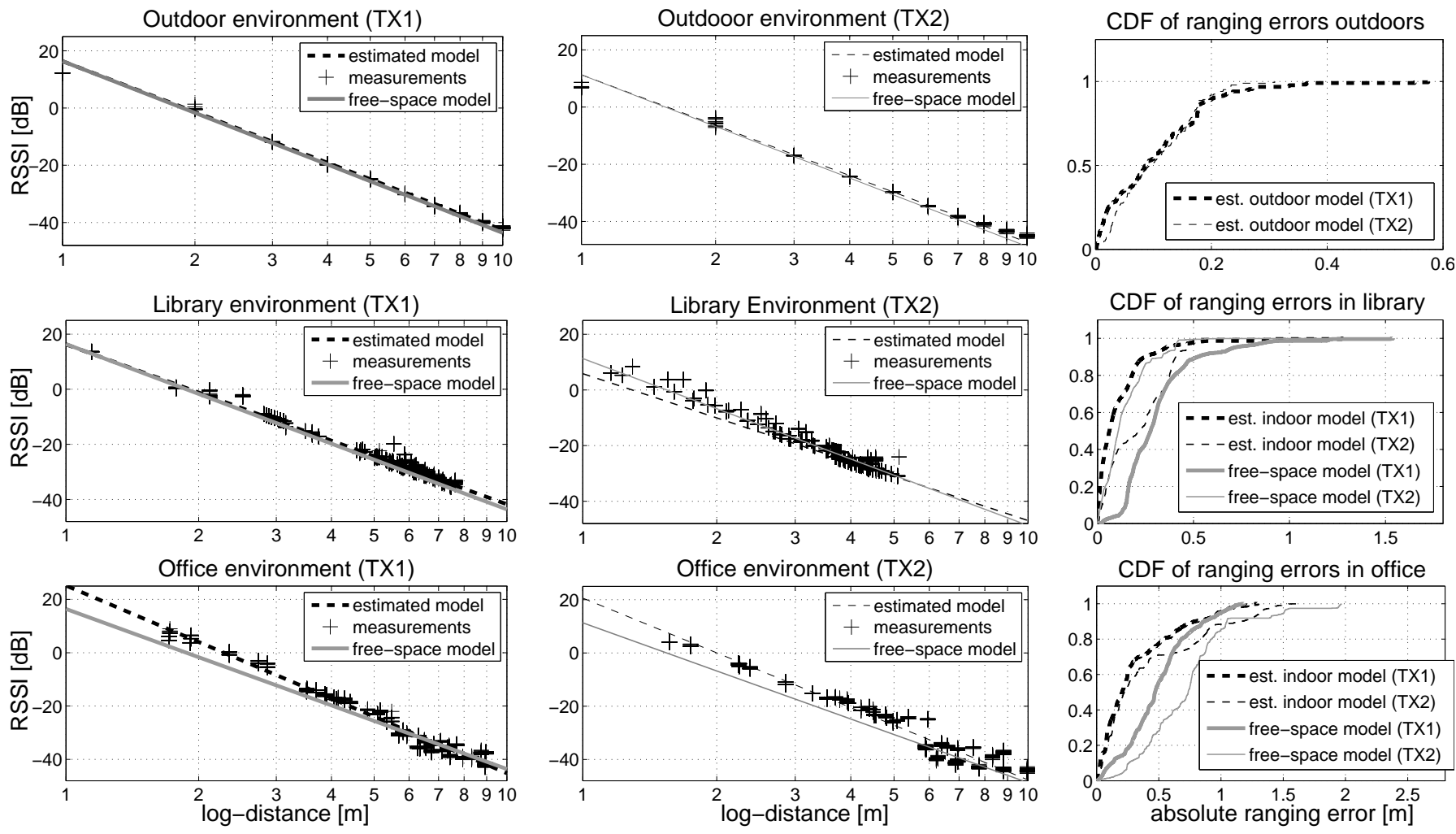

Fig. 6. Estimated path-loss model in different environments (outdoor - top row, library - middle row, office - bottom row) vs. theoretical free-space model (TX1 - left column, TX2 - middle column). The CDF of the ranging errors (right column) is shown for the estimated models, as well as for the case when the theoretical free-space model is used instead (the gray curves).

and less frequent use of the channel matrix (lower $P_{\text {useful }}$ ). Increasing the threshold value to $\left(a_{2}=0.1\right)$ decreases $P_{\mathrm{FA}}$, and more importantly, the channel use increases to one third of the time, with the price of slightly higher value of $P_{\text {miss }}$. Therefore, the criterion proves to be useful in practical scenarios, where sporadic good estimates help adjust the system performance in an opportunistic manner.

\section{B. Range estimation}

The following tests were designed to address the following questions: 1) How accurate is the ranging (i.e., the estimated TX-RX distance) in the absence/presence of metallic structures? 2) Are the readings obtained by the system accurate enough to distinguish between points located just few $\mathrm{cm}$ apart from each other? 3) Are the readings stable over time?

1) Ranging accuracy - Indoor vs. outdoor: This experiment aimed at testing the system ranging accuracy both indoors and outdoors, for distances ranging between $1 \mathrm{~m}$ and $10 \mathrm{~m}$. Each row in Fig. 6 corresponds to an experiment in a different environment, as follows: outdoors (top), library (middle), office environment (bottom). We performed tests with two different transmitters (TX1 and TX2). The corresponding path-loss models are shown in the first, and the second column of subplots in Fig. 6, respectively. The third column in Fig. 6 shows the CDF of the ranging errors corresponding to the estimated model, and the free-space model.

The first experiment was performed outdoors. We took measurements at 12 fixed positions on each circle of radius
$1-10 \mathrm{~m}$ (the angular separation is approximately $30^{\circ}$ ). Fig. 6 (top row) shows that the slope of the estimated model agrees extremely well with the theoretical free space model for both transmitters (left, middle). The CDF plot (right) shows that we achieve accuracy of less than $20 \mathrm{~cm}$ in $90 \%$ of the cases.

The second experiment was conducted in a university library. Fig. 6 (middle row) shows the relationship between log-distance and RSSI (left and middle plots). In this indoor environment, we observe a slight slope difference between the fitted and theoretical free-space model. However, the variance of the measurements remains very low, unlike for RFbased indoor channel models. Therefore, the number of points required to get a reasonable channel model is much lower (from our experiments, we found that 10 to 20 measurements are sufficient). The CDF subplot (right) shows that we can achieve errors less than $20 \mathrm{~cm}$ in $90 \%$ of the cases for TX1 and $50 \%$ for TX2. Even if the free-space model is used instead of the estimated model, the ranging errors remain below $50 \mathrm{~cm}$ in $90 \%$ of the cases, which is a remarkable feature of the MIbased ranging.

The third experiment was carried out in an office environment with area of size $11 \times 21 \mathrm{~m}$, which includes two rooms and a corridor wrapping around them. TXs were placed in the middle of each room, and the channel properties for each transmitter are shown in Fig. 6 (bottom row). The CDF subplot (right) shows that even in an environment with metallic induced distortions, we can achieve range accuracy better than $50 \mathrm{~cm}$ for up to $70 \%$ of the measurements. We also evaluate 
the ranging errors in the case when the theoretical free-space model was used instead of the estimated model. For TX1, the ranging errors remain below $60 \mathrm{~cm}$ in $80 \%$ of the cases, and for TX2, below $90 \mathrm{~cm}$ in $80 \%$ of the cases. In applications where collecting measurements in advance for the model is undesirable, the free-space channel model may be used as a reasonable starting model, and then learned during operation, in a SLAM-like fashion [11]-[14].

2) Ability to detect tiny displacements: This experiment was also conducted in the library. When the receiver is stationary, we can use magnetic signals to distinguish between locations extremely close to each other. In order to demonstrate this potential, we placed the receiver on top of 8 folders located next to each other on the same shelf, as shown in Fig. 7 (left). The displacements of $5 \mathrm{~cm}$ (as we move from one book to another) correspond to distance changes of $4 \mathrm{~cm}$ with respect to the transmitter. We were surprised to see that even these tiny changes in range are detectable based on RSSI only. Fig. 7 shows the distinct steps as we move from one folder to another, and the agreement between true and estimated ranges.
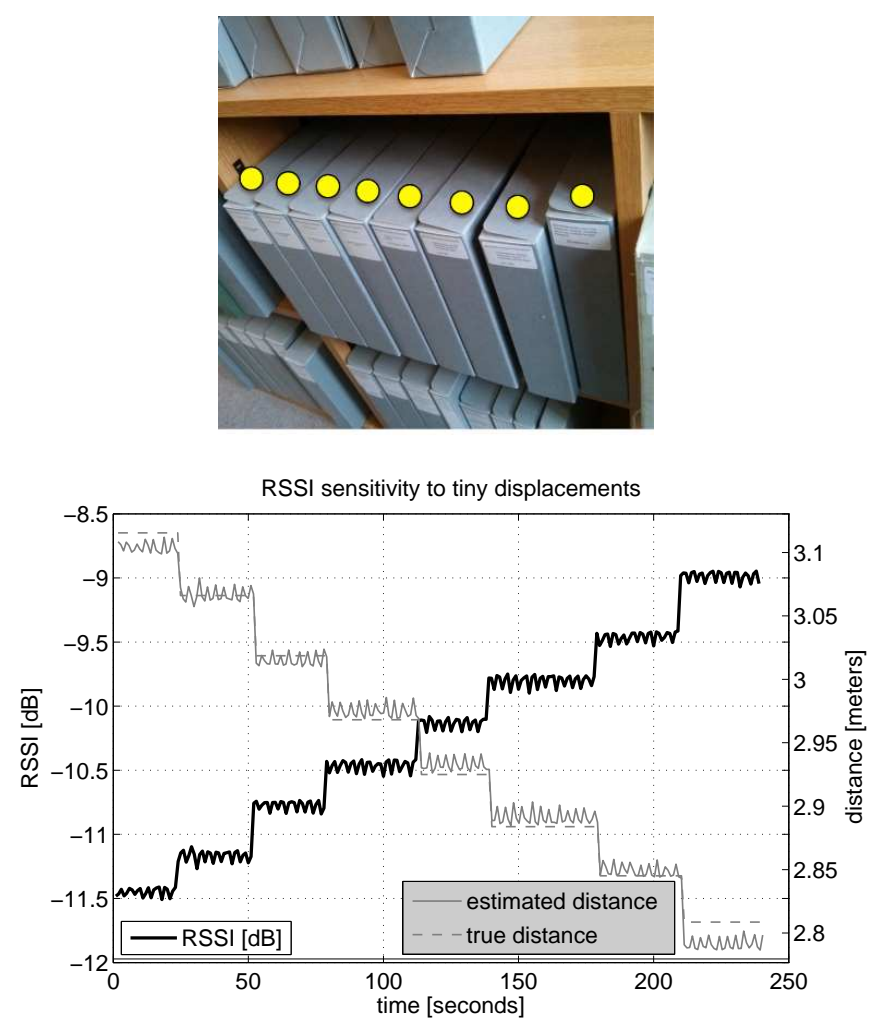

Fig. 7. Sensitivity of the signal strength to tiny receiver displacements (top) and ability to perform accurate ranging (bottom).

3) Time stability of the magnetic RSSI: The next experiment shows that the generated magnetic field strength is very stable over a long period of time, unlike the common RF signals. In Fig. 8, we show the variation of the RSSI of the magnetic link versus the RSSI corresponding to a WiFi link. RX and TX were stationary, placed at the same location in both cases, about $4 \mathrm{~m}$ apart, with TX at the origin. The RSSI was recorded for a period of approximately 8 minutes for each of the transceivers, while two persons were walking between TX and
RX, crossing the link, and approaching TX and RX. Fig.8 shows that the signal is extremely stable despite the fact that multiple people were passing by, and crossing line of sight to the transmitters. This is a unique advantage of the magnetic modality compared to radio-based techniques.
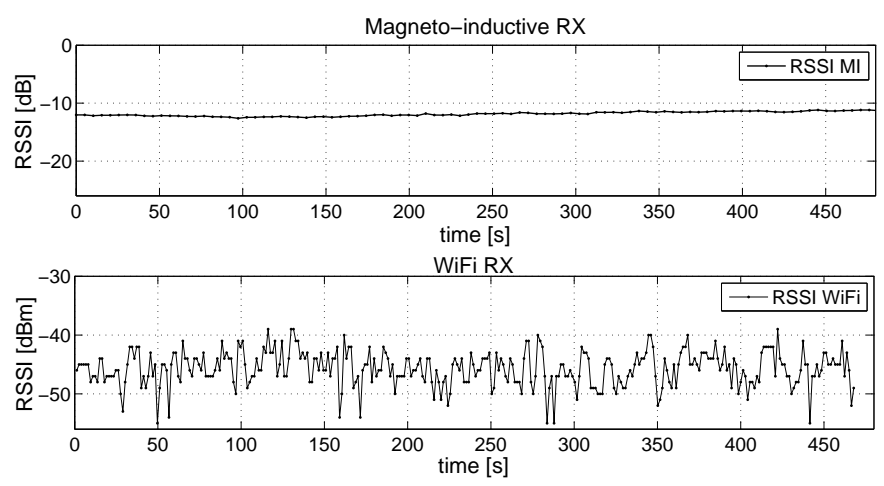

Fig. 8. The stability of magnetic signal strength vs. WiFi signal strength at a fixed location.

\section{Localization}

1) Outdoor localization: The goal of this experiment was to validate the 3-D position estimation algorithm for undistorted environments proposed in Section V-B. It was performed at a park along a semicircular path as shown in Fig. 9. A rope of length $5 \mathrm{~m}$ was tethered at Point $\mathrm{O}(2.5 \mathrm{~m}$ away from TX). A person holding the edge of the rope moved along the path at fast walking speed. The 3-D positions shown in Fig. 9 were estimated purely based on the magnetic field without any filtering. In this experiment, we did not fuse with inertial data because we wanted to test the position accuracy of the magnetic system alone. The mean absolute 3-D error in this experiment was $0.32 \mathrm{~m}$ and the standard deviation was $0.4 \mathrm{~m}$.

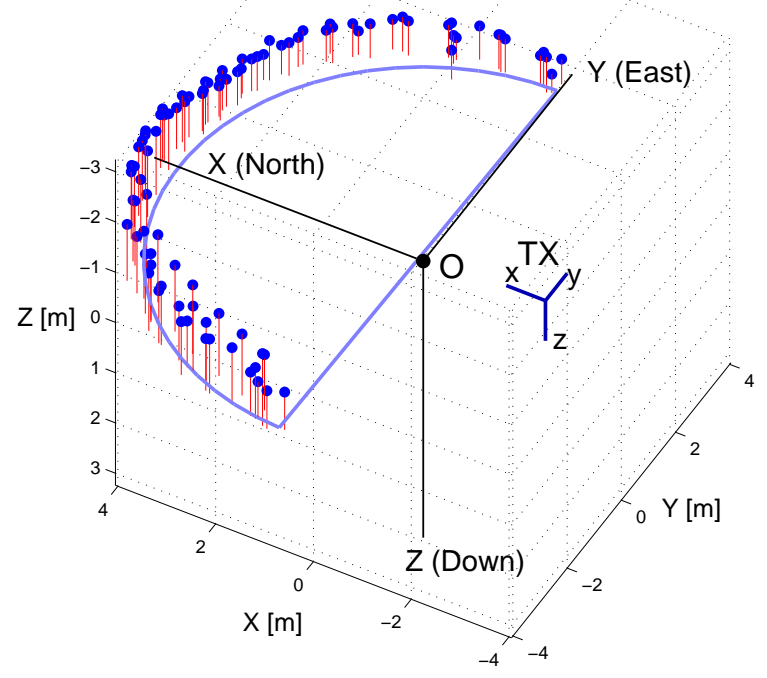

Fig. 9. Outdoor 3-D localization on the move. 


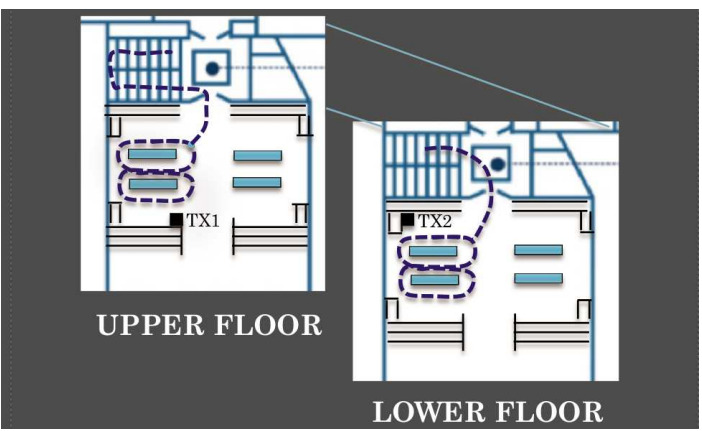

(a)

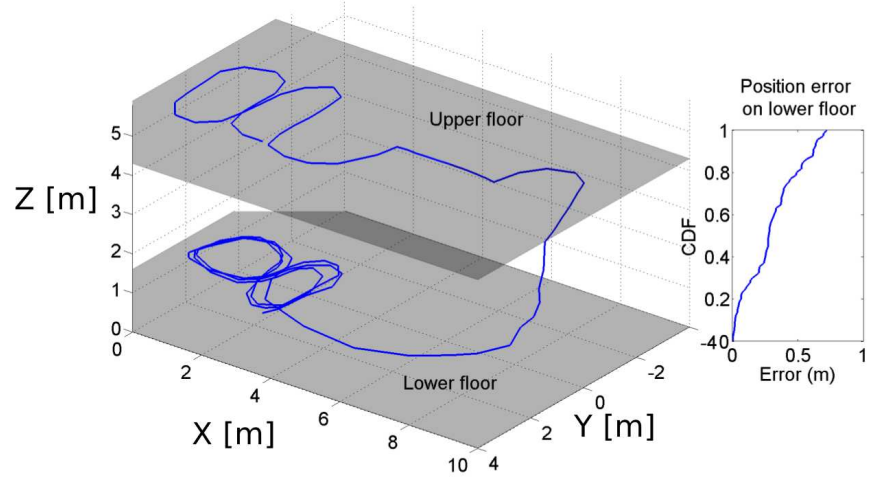

(b)

Fig. 10. Location tracking in library environment using modulated magnetic field and inertial sensors. (a) Library experiment site on two floors and ground truth. (b) Estimated 3-D Trajectory. The 8-shaped loop was performed once on the upper floor and three times on the lower floor.

2) Location Tracking in Multi-floor Building: In this section, we use our system to track the position of a user. The experiments were conducted on two levels of a university library; both levels house large collections of books and are also used as reading rooms. The floor-to-ceiling distance is $6.87 \mathrm{~m}$ for the upper level and $4.17 \mathrm{~m}$ for the lower level. Measurements were taken during a period of 3 hours, while people were crossing the test site. One transmitter was placed on the upper level (TX1) and another on the lower level (TX2), diagonally opposite, as shown in Fig. 10(a). Bookcases were placed both around and in the middle of the area, thus frequently blocking the line of sight from the transmitter to the receiver locations. Line of sight was also often blocked by the bodies of the people performing the experiments. The ground truth of the trajectory is shown in Fig. 10(a). A person holding MagLoc receiver followed an "8-shaped" trajectory around the book cases of the upper floor, came out of the reading room, went down the stairs, and repeated (three times) a similar "8-shaped" trajectory in the reading room of the lower floor. The following PDR parameters are used: $\sigma_{d}=0.5 \mathrm{~m}$, $\sigma_{\theta}=\pi / 8$, and $h_{s}=0.18 \mathrm{~m}$. The particle filter parameters are $\sigma_{r}=1.5 \mathrm{~m}$, and $\sigma_{\phi}=\pi / 8$. The number of particles is $10^{4}$. By fusing magnetic and inertial data only, and without using the building's floor-plan, we are able to obtain a 3-D trajectory as shown in Fig. 10(b). The mean error w.r.t. ground truth locations on the lower floor is $0.3 \mathrm{~m}$, and the standard deviation is $0.21 \mathrm{~m}$.

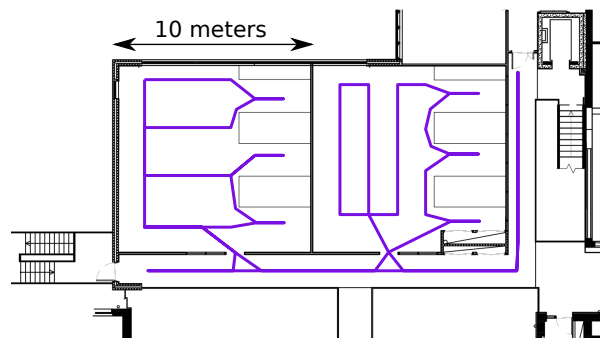

(a)

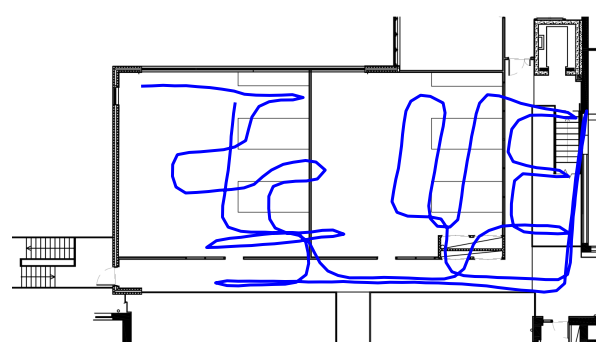

(b)

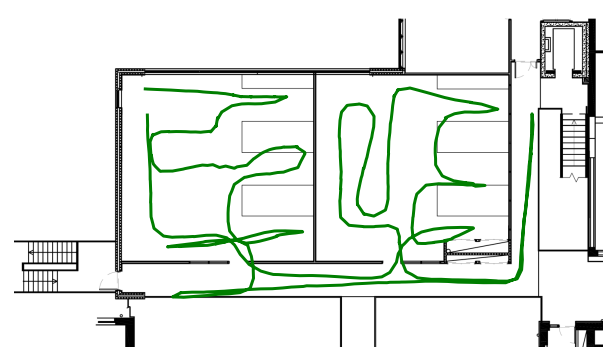

(c)

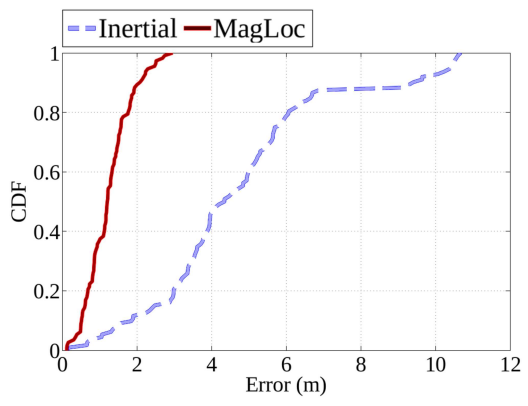

(d)

Fig. 11. Location tracking in office environment using modulated magnetic field and inertial sensors. (a) Ground truth. (b) The inertial only trajectory. (c) MagLoc using the magnetic channel model and the inertial data. (d) CDF of the positioning error.

3) Location Tracking in Office Environment: The last set of experiments was run in an office environment that is heavily reinforced with steel beams and mesh, and as such, the level of metallic induced distortion is very high. The ground truth of the trajectory is shown in Fig. 11(a). We tested the position accuracy of MagLoc system following tortuous trajectories around desks and other office furniture. Figs. 11(b) and 11(c) show the paths estimated using inertial data only, and 
using the proposed multi-layer fusion of inertial and magnetic data, respectively. In this study, we employ a particle filter, as described in Section V-E. Accuracy is measured against ground truth data from labeled positions, which are obtained from a mobile phone camera. Notice that by fusing magnetic and inertial data, we achieve a mean position accuracy of $0.8 \mathrm{~m}$ and a standard deviation $0.48 \mathrm{~m}$ without the need of a floor map, and without fingerprinting. The CDF of the position errors in both cases is shown in Fig. 11(d). Our sub-meter accuracy localization performance justifies the use of fixed infrastructure in many application where accuracy is crucial.

\section{BACKGROUND}

Radio-based positioning: There is a plethora of radio technologies for positioning in GPS-denied environments, including RFID, WiFi, BTLE, FM and UWB. RFID technology is mainly used for proximity sensing when tagged objects are in the vicinity of RFID readers. WiFi RSS-based systems have gained a lot of attention recently due to the wide availability of WiFi access points and smartphones. The first generation of WiFi systems relied on a radio propagation model to infer the distance between a transmitter (e.g. WiFi AP) and a receiver (e.g. smartphone) as a function of the transmitted and received signal strength. However, in practice, even sophisticated models are not sufficient to capture the complexity of WiFi signal propagation in challenging environments. This problem is partly addressed by the second generation of WiFibased systems, which is based on the idea of fingerprinting. Fingerprinting techniques, such as Radar [15] and Horus [16] typically involve two phases: In an initial training phase, they collect RSSI information from multiple APs at different locations of a building and build a radio map. In the second phase, they infer location by comparing the RSSI detected by a device with those stored in the radio map. Further techniques have been developed to deal with heterogeneous wireless clients [17] or to exploit additional features of the environment, e.g. FM signals [18], sound, light and color [19]. The key problem with fingerprinting approaches is the intensive surveying effort required to collect training data. The third generation of WiFibased systems addresses this problem by means of crowdsourcing WiFi and inertial data, and using automated zeroeffort fingerprinting techniques to build a radio map (see Fusion techniques below). Radio-interferometric localization techniques have also been proposed (e.g. [20]).

WiFi (and more recently BTLE) RSS-based positioning systems face a fundamental limitation arising from the properties of the signal that they employ. Specifically, the time variability of radio signals and the complex multi-path and attenuation effects due to fixed and moving obstacles prevent them from crossing the barrier of $2-3 \mathrm{~m}$ of accuracy in benign environments, and causes their severe degradation in more challenging environments. Time-delay based (ToA/RTT/TDOA) (e.g. [21], [22] and angle-of-arrival methods are more accurate, but they are still impaired by multi-path and/or non-line-ofsight effects in indoor environments. Ultrawideband (UWB) has enhanced ability to separate multipath contributions in line-of-sight conditions, but still suffers to some extent from dispersion/attenuation in non-line-of sight conditions. As it is a conventional trilateration approach, it requires good placement of non-colocated detectors, with a minimum of four receivers for 3-D tracking. For maximum accuracy, the UWB receivers need to be placed around the perimeter of the area to be tracked. However, this comes at a significant infrastructure cost and complexity. Infrastructure nodes must be synchronized exactly, requiring line of sight or timing cables, and must be placed carefully (distributed as evenly as possible and at elevated positions) to obtain good position accuracy and to avoid blind spots [23].

Motion sensing: This involves fusing data generated by IMUs to compute the user trajectory relative to her initial position. Some techniques assume that IMUs are mounted on the foot of the person [24]-[26], whereas others obtain data from IMUs embedded in consumer electronic devices, such as smartphones [27]. Inertial motion sensing is performed by iteratively repeating the following three tasks: 1) Motion Mode Recognition, which uses accelerometer data to distinguish between different modes of movement (e.g. static, walking and hand texting, walking with phone in a bag, etc.) [27]; 2) Orientation Tracking, which uses magnetometer, accelerometer and, optionally, gyroscope data to estimate the device orientation [28], [29]; 3) Step Length Estimation, which uses accelerometer data to estimate step length [30]. The last two tasks iteratively extend the trajectory by detecting a step, and extending the trajectory by the estimated length of that step, along the estimated orientation. Practical challenges in motion sensing, such as the sensitivity to phone position and the variability in user walking profiles, are explored in [31]. In cooperative scenarios, accuracy can further be improved by fusing inertial data with user encounter information [32], [33].

Magnetic-based positioning: In the past years, there has been a growing interest in localization systems using magnetic fields [3], [10], [34]-[38]. This is partly because cheap magnetic sensors are nowadays available in almost every handheld smart device. In addition, these systems usually operate at extremely low frequencies, in the near field region [5]. Consequently, the corresponding signals are non-propagating, and they do not experience multipath and shadow fading, such as the high frequency radio waves. Unlike microwaves, the magnetic fields experience no absorption by water (e.g. by the human body), which makes them very attractive for localization inside highly populated buildings, where people are continuously moving. Moreover, they do not usually require lineof-sight between devices, as they have the ability to penetrate through soil, concrete and rock with negligible attenuation [10]. Most existing magnetic localization approaches use either indigenous magnetic fields, such as the Earth's magnetic field and/or the magnetic fields generated by home electronics [36]-[38]. There are also approaches based on low-frequency magnetic fields generated locally for localization purposes [10], [34], [35]. In [10], the field was created by a grid of long coils which is impractical indoors. A very short range relative proximity detection platform (LiveSynergy) using near field MI devices equipped with 3-D coils was proposed in [39]. In [34] location was estimated under the assumption of controlled and known receiver orientation, which is not 
realistic for on-the-move localization. Unlike the work in [35] we do not have the need for time synchronization between transmitters and receivers, and triple the range through signal processing and an optimized receiver design, requiring a much sparser network. We would like to point out that the difference between the proposed MI and the plethora of existing geomagnetic localization systems. It is not just the magnetic field being generated locally, but there are several fundamental differences. First, we are using 3-D modulated vector field orientation to do 3-D positioning. We are using triaxial coils both at TX and RX, and this allows us to obtain a $3 \times 3$ channel matrix. This matrix enables the orinetation invariance property for both TX and RX. In addition, it leads to completely different 3-D position estimation algorithms. In Section V-B, we propose an elegant matrix solution for 3-D position estimation, which geomagnetic localization alone cannot achieve, even in undistorted environments, unless extra-sensors are used. For example, IndoorAtlas [37] exploits the distortions in the Earth's magnetic field, caused by the reinforced steel superstructure of modern buildings, to provide infrastructureless 2-D indoor positioning. However, the accuracy of these techniques is fundamentally limited by the spatial variability of the distortions in the magnetic field maps, making it unusable in undistorted environments such as outdoors. Moreover, they require the user to move before it is possible to converge to a potential 2-D position.

Fusion techniques: Most existing algorithms, such as Hidden Markov Models (HMMs), Kalman and Particle Filters, are typically based on Bayesian estimation. HMMs have been widely used for map matching and location estimation, both outdoors using road maps [40]-[42] as well as indoors [43], [44]. An alternative approach is to consider the location of a user as a continuous variable and resort to a Kalman Filter variant (e.g. [45]). Another common technique for data fusion and map matching is to use particle filters [8], [25], [31], [46], [47], or conditional random fields [6]. Other approaches such as WiFi-based SLAM fuse RSS and motion sensor data to simultaneously build a map of the environment and locate the user within this map [11]-[14]. More recent approaches focused on organic landmark maps and their use for positioning [48], [49]. Thanks to the stability properties of the magnetic channel, our novel MI positioning system is an ideal candidate for fusion with other sensor modalities, such as inertial data, as demonstrated in Section VII.

\section{CONCLUSiOnS}

The vision of localization is to have a simple to deploy, highly accurate and responsive 3 -D positioning system that is immune to non-line of sight issues caused by obstacles and moving people. An accurate and reliable positioning service is essential for a multitude of applications such as indoor location-based-advertising, assistive living, warehousing and robotics. MagLoc positioning system presented in this paper is a step towards this ideal, as it provides excellent positioning accuracy in 3-D from a single transmitter which can be hidden away in a convenient location. Not only is it able to track in 3$\mathrm{D}$, it also is able to operate over multiple floors through solid concrete, a major advantage over existing techniques. Through a process of optimization over the entire signal chain, from front-end topologies to correlation and fusion, we are able to overcome a number of limitations inherent to MagnetoInduction, and we believe that it is a strong contender to become a ubiquitous positioning solution. Although current mobile phones are unable to detect these magnetic fields due to insufficient bandwidth, analog magneto-resistive sensors exist which can operate up to $5 \mathrm{MHz}$. Thus, it is perfectly feasible that the next generation of smartphones could detect these fields, a goal we are working towards.

\section{REFERENCES}

[1] R. P. Feynman, R. B. Leighton, and M. Sands, The Feynman Lectures on Physics. Addison Wesley, 1964, vol. II.

[2] J. B. Kuipers, Quaternions and Rotation Sequences. A Primer with Applications to Orbits, Aerospace, and Virtual Reality. Princeton University Press, 1999.

[3] A. Markham and N. Trigoni, "Magneto-inductive networked rescue system (MINERS): taking sensor networks underground," in 11th International Conference on Information Processing in Sensor Networks (IPSN 2012), Beijing, China, Apr. 16-20 2012.

[4] K. Yamazaki and T. Kawamoto, "Simple estimation of equivalent magnetic dipole moment to characterize ELF magnetic fields generated by electric applicances incorporating harmonics," IEEE Transactions on Electromagnetic Compatibility, vol. 43, no. 2, 2001.

[5] J. I. Agbinya, Principles of Inductive Near Field Communications for Internet of Things, ser. River Publishers Series on Communications. River Publishers, 2011.

[6] Z. Xiao, H. Wen, A. Markham, and N. Trigoni, "Lightweight map matching for indoor localisation using conditional random fields," in Proceedings of the 13th International Symposium on Information Processing in Sensor Networks, IPSN-14, April 2014, pp. 131-142.

[7] I. Cleland, B. Kikhia, C. Nugent, A. Boytsov, J. Hallberg, K. Synnes, S. McClean, and D. Finlay, "Optimal placement of accelerometers for the detection of everyday activities," Sensors, vol. 13, no. 7, pp. 91839200, 2013.

[8] A. Rai, K. K. Chintalapudi, V. N. Padmanabhan, and R. Sen, "Zee: zero-effort crowdsourcing for indoor localization," in Mobicom, 2012.

[9] International Commission on Non-Ionizing Radiation Protection, "ICNIRP guidelines for limiting exposure to time-varying electro-magnetic fields (1 Hz-100 kHz)," Health Physics, vol. 99, no. 6, pp. 818-836, 2010.

[10] A. Markham, N. Trigoni, S. A. Ellwood, and D. W. Macdonald, "Revealing the hidden lives of underground animals using magnetoinductive tracking," in 8th ACM Conference on Embedded Networked Sensor Systems (Sensys 2010), Zürich, Switzerland, Nov. 2010.

[11] B. Ferris, D. Fox, and N. Lawrence, "WiFi-SLAM using Gaussian process latent variable models," in IJCAI, 2007.

[12] P. Robertson, M. Angermann, and M. Khider, "Improving simultaneous localization and mapping for pedestrian navigation and automatic mapping of buildings by using online human-based feature labeling," in PLANS, 2010

[13] P. Robertson, M. Angermann, B. Krach, and M. Khider, "Slam dance: Inertial-based joint mapping and positioning for pedestrian navigation," in Proc. Inside GNSS, 2010.

[14] Z. Yang, C. Wu, and Y. Liu, "Locating in fingerprint space: wireless indoor localization with little human intervention," in MobiCom, 2012.

[15] P. Bahl and V. Padmanabhan, "RADAR: an in-building RF-based user location and tracking system," in INFOCOM, 2000.

[16] M. Youssef and A. Agrawala, "The Horus WLAN location determination system," in MobiSys, 2005.

[17] M. B. Kjaergaard, "Indoor location fingerprinting with heterogeneous clients," Pervasive Mob. Comput., vol. 7, no. 1, pp. 31-43, 2011.

[18] Y. Chen, D. Lymberopoulos, J. Liu, and B. Priyantha, "FM-based indoor localization," in Mobisys, 2012, pp. 169-182.

[19] M. Azizyan, I. Constandache, and R. Roy Choudhury, "SurroundSense: mobile phone localization via ambience fingerprinting," in MobiCom, 2009.

[20] I. Amundson, J. Sallai, X. Koutsoukos, and A. Ledeczi, "Radio interferometric angle of arrival estimation," in The 7th European Conference on Wireless Sensor Networks. Coimbra, Portugal: Springer, Feb. 2010. 
[21] A. Günther and C. Hoene, "Measuring round trip times to determine the distance between WLAN nodes," NETWORKING 2005, pp. 768779, 2005.

[22] T. E. Abrudan, A. Haghparast, and V. Koivunen, "Time-synchronization and ranging for OFDM systems using time-reversal," IEEE Transactions on Instrumentation and Measurement, vol. 62, no. 12, pp. 3276-3290, Dec. 2013.

[23] E. Mok, F. Lau, L. Xia, G. Retscher, and H. Tian, "Influential factors for decimetre level positioning using ultra wide band technology," Survey Review, vol. 44, no. 324, pp. 37-44, 2012.

[24] E. Foxlin, "Pedestrian tracking with shoe-mounted inertial sensors," in IEEE Com. Graph. App., 2005

[25] O. Woodman and R. Harle, "Pedestrian localisation for indoor environments," in UbiComp, 2008.

[26] A. Jimenez, F. Seco, C. Prieto, and J. Guevara, "A comparison of pedestrian dead-reckoning algorithms using a low-cost MEMS IMU,' in WISP, 2009.

[27] M. Susi, V. Renaudin, and G. Lachapelle, "Motion mode recognition and step detection algorithms for mobile phone users," Sensors, vol. 13, no. 2, 2013.

[28] B. Huyghe, J. Doutreloigne, and J. Vanfleteren, “3D orientation tracking based on unscented Kalman filtering of accelerometer and magnetometer data," in Proc. SAS, 2009.

[29] Y. S. Suh, "Orientation estimation using a quaternion-based indirect Kalman filter with adaptive estimation of external acceleration," IEEE Transactions on Instrumentation and Measurement, vol. 59, no. 12, 2010.

[30] V. Renaudin, M. Susi, and G. Lachapelle, "Step length estimation using handheld inertial sensors," Sensors, vol. 12, no. 7, pp. 8507-8525, 2012.

[31] F. Li, C. Zhao, G. Ding, J. Gong, C. Liu, and F. Zhao, "A reliable and accurate indoor localization method using phone inertial sensors," in UbiComp, 2012

[32] I. Constandache, X. Bao, M. Azizyan, and R. R. Choudhury, "Did you see Bob?: human localization using mobile phones," in MobiCom, 2010.

[33] A. Symington and N. Trigoni, "Encounter based sensor tracking," in MobiHoc, 2012

[34] A. Sheinker, B. Ginzburg, N. Salomonski, L. Frumkis, and B. Kaplan, "Localization in 3-D using beacons of low frequency magnetic field," IEEE Transactions on Instrumentation and Measurement, vol. 62, 2013.

[35] G. Pirkl and P. Lukowicz, "Robust, low cost indoor positioning using magnetic resonant coupling," in ACM International Joint Conference on Pervasive and Ubiquitous Computing (UbiComp 2012), 2012.

[36] M. Frassl, M. Angermann, M. Lichtenstern, P. Robertson, B. J. Julian, Marek, and Doniec, "Magnetic maps of indoor environments for precise localization of legged and non-legged locomotion," in Proceedings of the IEEE/RSJ International Conference on Intelligent Robots and Systems (IROS 2013), Tokyo, Japan, Nov. 2013

[37] J. Haverinen and A. Kemppainen, "Global indoor self-localization based on the ambient magnetic field," Robotics and Autonomous Systems, vol. 57, pp. 1028-1035, 2009.

[38] S. Rahok and O. Koichi, "Odometry correction with localization based on land-markless magnetic map for navigation system of indoor mobile robot," in 4th International Conference on Autonomous Robots and Agents (ICARA 2009), Feb. 2009, pp. 572-577.

[39] X. Jiang, C.-J. M. Liang, K. Chen, B. Zhang, J. Hsu, J. Liu, B. Cao, and F. Zhao, "Design and evaluation of a wireless magnetic-based proximity detection platform for indoor applications," in IPSN. Association for Computing Machinery (ACM), April 2012.

[40] A. Thiagarajan, L. Ravindranath, K. LaCurts, S. Madden, H. Balakrishnan, S. Toledo, and J. Eriksson, "Vtrack: accurate, energy-aware road traffic delay estimation using mobile phones," in SenSys, 2009.

[41] P. Newson and J. Krumm, "Hidden Markov map matching through noise and sparseness," in SIGSPATIAL GIS, 2009.

[42] S. Guha, K. Plarre, D. Lissner, S. Mitra, B. Krishna, P. Dutta, and S. Kumar, "AutoWitness: locating and tracking stolen property while tolerating GPS and radio outages," in SenSys, 2010.

[43] J. Seitz, T. Vaupel, J. Jahn, S. Meyer, J. G. Boronat, and J. Thielecke, "A hidden Markov model for urban navigation based on fingerprinting and pedestrian dead reckoning." in FUSION, 2010.

[44] H. Wen, Z. Xiao, N. Trigoni, and P. Blunsom, "On assessing the accuracy of positioning systems in indoor environments," in EWSN, 2013.

[45] W. Chai, C. Chen, E. Edwan, J. Zhang, and O. Loffeld, "INS/Wi-Fi based indoor navigation using adaptive Kalman filtering and vehicle constraints," in WPNC, 2012

[46] S. Arulampalam, S. Maskell, N. Gordon, and T. Clapp, "A tutorial on particle filters for online nonlinear / non-Gaussian Bayesian tracking,"
IEEE Transactions on Signal Processing, vol. 50, no. 2, pp. 174-188, 2002.

[47] S. Beauregard, Widyawan, and M. Klepal, "Indoor PDR performance enhancement using minimal map information and particle filters," in PLANS, 2008

[48] H. Wang, S. Sen, A. Elgohary, M. Farid, M. Youssef, and R. R. Choudhury, "No need to war-drive: unsupervised indoor localization," in MobiSys, 2012.

[49] G. Shen, Z. Chen, P. Zhang, T. Moscibroda, and Y. Zhang, "WalkieMarkie: indoor pathway mapping made easy," in NSDI, 2013.

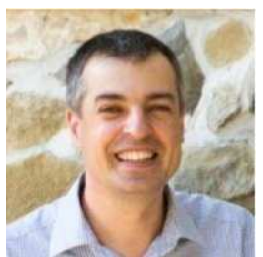

Traian E. Abrudan (S'02-M'09) received the D.Sc. degree (with honors) from Aalto University, Finland (2008), and the M.Sc. degree from the Technical University of Cluj-Napoca, Romania (2000). Since 2013, he has been a postdoctoral researcher at the Department of Computer Science, University of Oxford, working on practical localization algorithms and systems for humans and robots using lowfrequency magnetic fields, as well as other sensing modalities. His fundamental research topics include sensor array signal processing, applied parameter estimation, numerical optimization, and wireless transceiver algorithms. During 2010-2013, he was a postdoctoral researcher at the Faculty of Engineering, University of Porto, and a member of Instituto de Telecomunicações, Portugal.

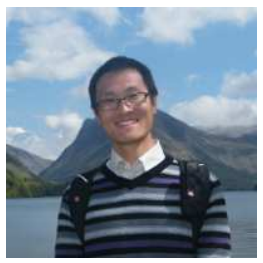

Zhuoling Xiao (S'14) is currently a $\mathrm{PhD}$ candidate in Computer Science Department at University of Oxford. His research interests focus on sensor networks, including localization, communication and coordination protocols for networked sensor nodes, and machine learning techniques for sensor networks and localization.

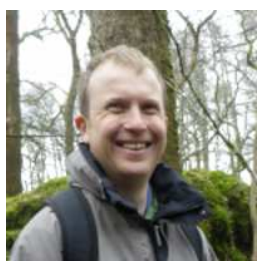

Andrew Markham received the Bachelor's (2004) and PhD (2008) degrees in Electrical Engineering from the University of Cape Town, South Africa. $\mathrm{He}$ is currently an Associate Professor in the Department of Computer Science, at the University of Oxford, working in the Sensor Networks Group. His research interests include low power sensing, embedded systems and magneto-inductive techniques for positioning and communication.

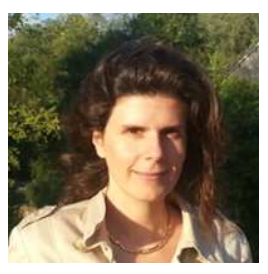

Dr. Niki Trigoni is an Associate Professor at the Department of Computer Science, University of Oxford. She obtained her $\mathrm{PhD}$ at the University of Cambridge (2001), became a postdoctoral researcher at Cornell University (2002-2004), and a Lecturer at Birkbeck College (2004-2007). Since she moved to Oxford in 2007, she established the Sensor Networks Group, and has conducted research in communication, localization and in-network processing algorithms for sensor networks. Her recent and ongoing projects span a wide variety of sensor networks applications, including indoor/underground localization, wildlife sensing, road traffic monitoring, autonomous (aerial and ground) vehicles, and sensor networks for industrial processes. 